Revista de Psicología y Educación / Journal of Psychology and Education, 2020, 15(1), 1-15 (www.rpye.es) Doi: https://doi.org/10.23923/rpye2020.01.182

ISSN: $1699-9517$

\title{
Predictores de ser víctima de acoso escolar en Educación Primaria: una revisión sistemática
}

\author{
Zara Suárez-García*, David Álvarez-García y Celestino Rodríguez \\ Universidad de Oviedo
}

\begin{abstract}
Resumen: El acoso escolar conlleva importantes consecuencias negativas para las víctimas. Por ello, es fundamental desarrollar medidas para su prevención o, en su caso, detección temprana. Para ello es imprescindible identificar sus principales factores de riesgo o protección, a partir de la evidencia científica disponible. El presente trabajo tiene como objetivo identificar los principales predictores de ser víctima de acoso escolar en Educación Primaria, a partir de una revisión sistemática de los artículos empíricos más relevantes publicados en la última década. Para ello, se realizó una búsqueda en las bases de publicaciones Psyclnfo, Eric, Web of Science y EbscoHost. Como resultado, se obtuvieron 49 artículos que cumplieron los criterios de búsqueda. Estos artículos destacan la capacidad predictiva de diversas variables individuales, escolares, familiares y comunitarias. Se discuten las implicaciones prácticas de estos resultados.
\end{abstract}

Palabras clave: Víctima, Acoso Escolar, Predictores, Educación Primaria, Revisión Sistemática.

\section{Predictors of being a victim of Bullying in Primary Education: a systematic review}

\begin{abstract}
Bullying has important negative consequences for victims. Therefore, it is essential to develop measures for their prevention or, where appropriate, early detection. In this sense and based on the available scientific evidence, it is essential to identify its main risk factor or protection variables. Main objective of this study is to identify the main predictors of being a victim of bullying in Primary Education, based on a systematic review of the most relevant empirical articles published in the last decade. For this, a search was made in the publications data bases of Psyclnfo, Eric, Web of Science and EbscoHost. As a result, 49 articles were obtained that met the search criteria. These articles highlight the predictive capacity of individual, school, family and community variables. The practical implications of these results are discussed.

Keywords: Victim, Bullying, Predictors, Primary Education, Systematic Review.
\end{abstract}

Los centros educativos son un contexto clave para el desarrollo socioafectivo de niños y adolescentes. En general, el clima escolar es bueno y favorece un desarrollo saludable (García-Bacete, Sureda, y Monjas, 2010). Sin embargo, en ocasiones el ambiente de convivencia no es satisfactorio y algunos alumnos son víctimas de agresiones. Un tipo de agresión especialmente grave es el acoso escolar, que se refiere a situaciones en las

Recibido: 24/04/2019 - Aceptado: 08/05/2019 - Avance online: 26/07/2019 *Correspondencia: Zara Suárez García.

Universidad de Oviedo.

C.P: 33003, Oviedo, España.

E-mail:vo186794@uniovi.es

Suárez-García, Z., Álvarez-García, D. y Rodríguez, C. (Avance Online). Predictores de ser víctima de acoso escolar en Educación Primaria: una revisión sistemática. Revista de Psicología y Educación, 15(1), 1-15, hitps://doi.org/10.23923/rpye2020.01.182 que uno o varios agresores ejercen diferentes tipos de violencia hacia una víctima (por ej. insultos, amenazas o agresiones físicas), de manera continuada en el tiempo y en las que la víctima está en inferioridad respecto a los agresores (por timidez, escasas habilidades sociales o falta de apoyo de sus compañeros) (Olweus, 1993).

El acoso escolar es un problema digno de atención por su prevalencia y efectos. Con respecto a su prevalencia, diversos estudios sitúan entre el $10 \%$ y el $33 \%$ el porcentaje de alumnado que es víctima de bullying (Hymel y Swearer, 2015). Con respecto a sus consecuencias, ser víctima de acoso escolar 
puede dar lugar a síntomas depresivos (Estévez et al., 2009; Graham et al., 2006; Jankauskiene et al., 2008; Kerr et al., 2011) y de ansiedad (Yang et al., 2013), y con ello a un descenso del rendimiento académico (Rettew y Pawlowski, 2016). En los casos más graves, el acoso escolar puede dar lugar a ideación suicida, intentos de suicidio o incluso suicidios consumados (Brunstein, Sourander y Gould, 2010).

Por la gravedad de este problema, resulta fundamental tratar de prevenir su aparición, ya desde las primeras etapas educativas. Para ello, es necesario identificar las variables que permiten predecir su aparición, como factores de riesgo o de protección. En los últimos años el interés y el número de trabajos referidos a este tema han aumentado notablemente. Es importante, por lo tanto, contar con revisiones sistemáticas actualizadas que permitan organizar y sintetizar sus principales hallazgos. En esta década, ya se han publicado algunas revisiones sistemáticas en este sentido (Cook, et al., 2010; Espelage, 2014; Kljakovic y Hunt, 2016; López et al., 2011 ; Saarento et al., 2014), que sin duda han supuesto una contribución al conocimiento y difusión de los predictores de ser víctima de acoso escolar. Sin embargo, hasta donde conocemos, ninguna de las revisiones previamente publicadas se centra específicamente en el rango de edad de la Educación Primaria en España (6-12 años). Esta etapa es fundamental en el desarrollo socioafectivo del alumnado y es clave en la prevención e identificación precoz del acoso escolar.

Por todo ello, el presente trabajo tiene el objetivo de identificar los principales predictores de ser víctima de acoso escolar en Educación Primaria, a partir de una revisión sistemática de los artículos empíricos más relevantes publicados en la última década.

\section{MÉTODO}

\section{PROCEDIMIENTO}

El proceso de selección de la muestra de los artículos analizados se presenta de forma resumida en la Figura 1. En marzo de 2018 se hizo una consulta en las bases de datos Psyclnfo, Eric, Web of Science y EbscoHost, utilizando como términos de búsqueda: (bullied OR "bullying victimization" OR "victim* of bullying") AND ("Primary education" OR "Primary school" OR "Elementary school" OR "Middle school") AND (Predictor* OR "Risk factor*" OR "Protective factor"). En Psyclnfo y Eric se limitaron los resultados a "Evaluados por Expertos"; en la Web of Science a "artículos"; y en EbscoHost a "publicaciones arbitradas". En todas las bases se restringió la búsqueda al periodo 2008-2018.

Los resultados de la búsqueda se introdujeron en el gestor bibliográfico Zotero y se eliminaron los duplicados. Posteriormente, a partir de los títulos y resúmenes, se hizo una selección de las publicaciones que cumplían los siguientes criterios: trabajos empíricos, referidos a predictores de ser víctima de bullying, con una muestra mayor de 500 sujetos y comprendida entre los 6 y los 12 años. Finalmente, se revisaron los textos completos con lo que se obtuvo la muestra final.

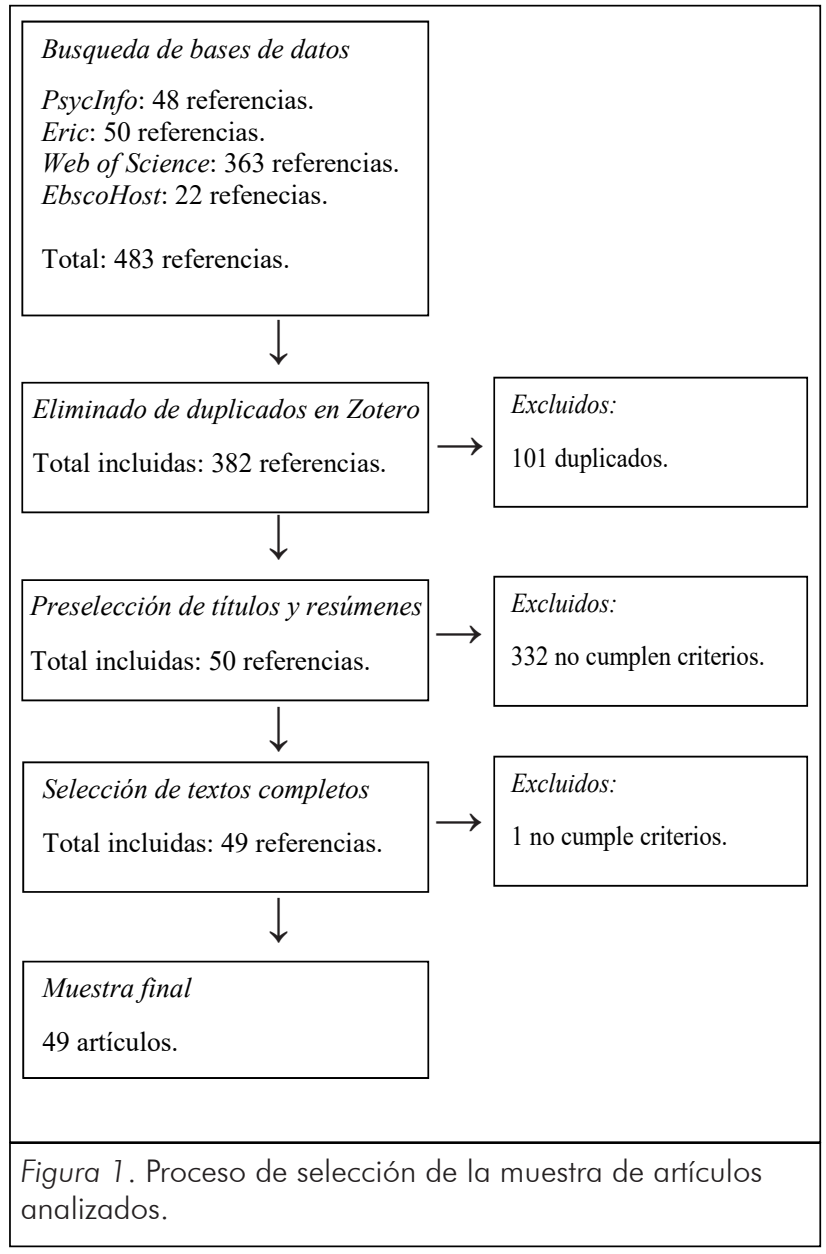


Para extraer la información relevante de los textos completos, se utilizó una plantilla previamente utilizada por Álvarez-García, García y Núñez (2015). En esta plantilla se anotó, para cada artículo, el año de publicación, la revista en la que fue publicado, el idioma, el número de estudiantes analizados, el rango de edad de la muestra, el país de la muestra, quién fue el informante, el método de recolección de datos y los principales resultados referidos a los predictores estudiados. Debido a la dificultad en la comprensión del idioma, uno de los artículos (Lee et al. 2014) sólo se utilizó para el análisis de las características bibliométricas y metodológicas y no para el análisis de predictores.

\section{MUESTRA}

Los 49 artículos revisados se publicaron en 32 revistas científicas diferentes (Tabla 1). Existe una tendencia a un aumento de publicaciones en los últimos 5 años. Los trabajos revisados han sido publicados en su gran mayoría en inglés.

En cuanto a las muestras de los estudios analizados (Tabla 2), el número de estudiantes evaluados oscila entre 512 y 22178, predominando las muestras comprendidas entre los 501 y 2000 participantes (63.2\%). Las edades más estudiadas, dentro del rango de 6 a 12 años considerado en esta revisión, son a partir de los 10 años. Los países a los que pertenecen

Tabla 1

Propiedades bibliométricas de los artículos incluidos en la revisión sistemática ( $N=49$ )

\begin{tabular}{|c|c|c|}
\hline Variable descriptiva & $f$ & $\%$ \\
\hline \multicolumn{3}{|l|}{ Año publicación } \\
\hline 2008 & 2 & 4.1 \\
\hline 2009 & 2 & 4.1 \\
\hline 2010 & 3 & 6.1 \\
\hline 2011 & 3 & 6.1 \\
\hline 2012 & 4 & 8.2 \\
\hline 2013 & 5 & 10.2 \\
\hline 2014 & 6 & 12.2 \\
\hline 2015 & 8 & 16.3 \\
\hline 2016 & 5 & 10.2 \\
\hline 2017 & 10 & 20.4 \\
\hline 2018 & 1 & 2.0 \\
\hline \multicolumn{3}{|l|}{ Idioma } \\
\hline Inglés & 46 & 93.9 \\
\hline Español & 1 & 2.0 \\
\hline Chino & 1 & 2.0 \\
\hline Coreano & 1 & 2.0 \\
\hline \multicolumn{3}{|l|}{ Revista } \\
\hline Journal of Youth Adolescence & 5 & 10.2 \\
\hline Aggressive Behavior & 3 & 6.1 \\
\hline Development Psychology & 3 & 6.1 \\
\hline Psicothema & 3 & 6.1 \\
\hline BMC Public Health & 2 & 4.1 \\
\hline Child Abuse \& Neglect & 2 & 4.1 \\
\hline Children and Youth of Clinical Psychology & 2 & 4.1 \\
\hline Journal of Abnormal Child Psychology & 2 & 4.1 \\
\hline Journal of Clinical Child and Adolescent Psychology & 2 & 4.1 \\
\hline School Psychology International & 2 & 4.1 \\
\hline School Psychology Quarterly & 2 & 4.1 \\
\hline Otros (un solo artículo) & 21 & 42.8 \\
\hline Total & 49 & 100.0 \\
\hline
\end{tabular}


las muestras son muy diversos, se encuentran en los cinco continentes, aunque mayoritariamente son de América del Norte (EEUU y Canadá) y posteriormente Europa (Holanda, Reino Unido, España y Finlandia).
Los artículos analizados usan diferentes informantes y métodos de recogida de información. Sin embargo, la gran mayoría utiliza cuestionarios de autoinforme (Tabla 3). En el $100 \%$ de los trabajos revisados se usan cuestionarios y en el $91.7 \%$ son de autoinforme.

Tabla 2

Características metodológicas de los artículos incluidos en la revisión sistemática (N=49), en referencia a la muestra analizada.

\begin{tabular}{|c|c|c|}
\hline Variable descriptiva & $f$ & $\%$ \\
\hline \multicolumn{3}{|l|}{ Número de niños analizados ${ }^{a}$} \\
\hline $501-1000$ & 18 & 36.7 \\
\hline $1001-2000$ & 13 & 26.5 \\
\hline $2001-3000$ & 4 & 8.1 \\
\hline $3001-4000$ & 3 & 6.1 \\
\hline $4001-5000$ & 2 & 4.1 \\
\hline $5001-10000$ & 6 & 12.2 \\
\hline$>10000$ & 3 & 6.1 \\
\hline \multicolumn{3}{|l|}{ Edad de la muestrab } \\
\hline Menos de 6 & 3 & 6.1 \\
\hline 6 & 8 & 16.3 \\
\hline 7 & 12 & 24.5 \\
\hline 8 & 13 & 26.5 \\
\hline 9 & 19 & 38.8 \\
\hline 10 & 35 & 71.1 \\
\hline 11 & 33 & 67.3 \\
\hline 12 & 31 & 63.3 \\
\hline Más de 12 & 23 & 46.9 \\
\hline \multicolumn{3}{|l|}{ País de la muestra } \\
\hline EEUU & 16 & 32.7 \\
\hline Canadá & 5 & 10.2 \\
\hline Holanda & 5 & 10.2 \\
\hline Reino Unido & 4 & 8.2 \\
\hline España & 3 & 6.1 \\
\hline Finlandia & 3 & 6.1 \\
\hline China & 2 & 4.1 \\
\hline Corea del Sur & 2 & 4.1 \\
\hline Alemania & 1 & 2.0 \\
\hline Australia & 1 & 2.0 \\
\hline Brasil & 1 & 2.0 \\
\hline Chile & 1 & 2.0 \\
\hline Israel & 1 & 2.0 \\
\hline Malawi & 1 & 2.0 \\
\hline República Checa & 1 & 2.0 \\
\hline Turquía & 1 & 2.0 \\
\hline Uganda & 1 & 2.0 \\
\hline Total & 49 & 100.0 \\
\hline
\end{tabular}


Tabla 3

Características metodológicas de los artículos incluidos en la revisión sistemática (N=49), relativas a la recogida de datos.

\begin{tabular}{|c|c|c|}
\hline Variable descriptiva & $f$ & $\%$ \\
\hline \multicolumn{3}{|l|}{ Informante } \\
\hline Autoinforme & 25 & 51.0 \\
\hline Autoinforme e informe de padres & 8 & 16.3 \\
\hline $\begin{array}{l}\text { Autoinforme, informe del profesor e informe de } \\
\text { pares }\end{array}$ & 3 & 6.1 \\
\hline Autoinforme, informe de padres e informe de pares & 3 & 6.1 \\
\hline $\begin{array}{l}\text { Autoinforme informe de padres e informe del } \\
\text { profesor }\end{array}$ & 3 & 6.1 \\
\hline Autoinforme e informe del profesor & 2 & 4.1 \\
\hline Autoinforme e informe de pares & 1 & 2.0 \\
\hline Informe padres e informe de pares & 1 & 2.0 \\
\hline Informe de padres e informe de profesor & 1 & 2.0 \\
\hline Informe de padres & 1 & 2.0 \\
\hline Informe de profesor & 1 & 2.0 \\
\hline \multicolumn{3}{|l|}{ Método de recolección de datos } \\
\hline Cuestionarios & 31 & 63.3 \\
\hline Cuestionarios y nominaciones de pares & 6 & 12.2 \\
\hline Cuestionarios y entrevista & 5 & 10.2 \\
\hline Cuestionarios y datos de bases estadísticas & 3 & 6.1 \\
\hline Cuestionarios y registros escolares & 2 & 4.1 \\
\hline Cuestionarios, nominaciones entre pares y entrevista & 2 & 4.1 \\
\hline Total & 49 & 100.0 \\
\hline
\end{tabular}

\section{RESULTADOS}

\section{FACTORES INDIVIDUALES}

\section{FACTORES SOCIODEMOGRÁFICOS}

En la mayoría de los trabajos que se analiza el sexo como variable predictora se halla que los niños son más susceptibles de ser víctimas de bullying que las niñas (Alcántara et al., 2017; Bowes et al., 2013; Cillenssen y Lansu, 2015; Foster y Brooks-Gunn 2013; García, Romera, y Ortega, 2015; Goldbach, Sterzing y Stuart, 2017; Han, Zhang y Zhang, 2017; Jansen et al., 2011; Jansen et al., 2012; Kollerova y Smolik, 2016; Pouwelse et al., 2011; Von Marées y Petermann, 2010; Watt et al., 2015). No obstante, algunos trabajos concluyen que las niñas son más víctimas (Wolke, Woods y
Samara, 2009), principalmente de violencia de tipo relacional (Jansen et al., 2012) y emocional (Wandera et al., 2017), e incluso otros estudios determinan que ser niño o niña no es predictor de ser víctima (Wang et al., 2014). Se ha analizado si es más probable ser víctima de acoso por parte de iguales del mismo sexo o del contrario, obteniendo resultados encontrados (Saarento et al., 2013; Veldkamp et al., 2017).

Con respecto a la edad, el alumnado con menos años tiene mayor probabilidad de ser víctima (Guerra, Williams y Sadek, 2011; Seo et al., 2017; Wandera et al., 2017). Además, en Primaria existe mayor tasa de intimidación y victimización frente a etapas educativas superiores (Han, Zhang y Zhang, 2017; Jansen et al., 2012). 
Todas las investigaciones que han estudiado la raza u origen étnico han tenido lugar en EEUU. Los resultados son poco concluyentes: Bradshaw, Sawyer y O'Brennan (2009) y Goldbach, Sterzing, y Stuart (2017) hallan que ser afroamericano o hispano es un factor de riesgo de ser víctima. Foster y Jeanne (2013) asociaron la victimización a ser blanco y Goldbach, Sterzing, y Stuart (2017) a ser caucásico, indio, hispano, asiático o de cualquier isla del pacífico.

\section{FACTORES FÍSICOS}

El aspecto físico y la capacidad física son predictores de ser víctima de acoso. La discapacidad física correlaciona con ser víctima de violencia emocional (Wandera et al., 2017). En este sentido, los niños motóricamente inferiores en preescolar tienen mayor probabilidad de ser víctimas en Primaria, mientras que los motóricamente más hábiles de ser agresores (Jansen et al, $2011)$. La obesidad también es un factor de riesgo de ser acosado (Lumeng et al., 2010).

\section{FACTORES PSICOLÓGICOS}

\section{- CAPACIDAD INTELECTUAL}

Déficits en la capacidad intelectual constituyen un factor de riesgo de padecer acoso escolar. En este sentido, tener un Cociente Intelectual bajo aumenta significativamente la probabilidad de sufrir bullying (Verlinden et al., 2014), al igual que sufrir una discapacidad intelectual (Wandera et al., 2017). Poseer unas habilidades cognitivas elevadas en edad preescolar es un factor protector frente a la victimización años después, ya en Educación Primaria (Watt et al., 2015).

\section{- RASGOS DE LA PERSONALIDAD}

- Problemas internalizados.

Puntuar alto en internalización de problemas está relacionado con ser intimidado (Bowes et al., 2013; Brendgen et al., 2016; Cillenssen y Lansu, 2015). Los síntomas depresivos (tristeza, autoestima baja, somatización) se asocian a una mayor probabilidad de ser víctima (Ameli et al., 2017; Berger-Silva, 2012; García, Romera y Ortega, 2015; Glew et al., 2008; Goldbach, Sterzing, y Stuart, 2017; Guerra, Williams y Sadek, 2011; Haltigan y Vaillancourt, 2014; Jansen et al., 2011 ; Moses y Williford, 2017; Pouwelse et al., 2011; Saarento et al., 2013; Sainio et al., 2012; Seo et al., 2017; Wandera et al., 2017; Williford, Boulton, y Jenson, 2014). Estos síntomas pueden deberse a victimización previa (Schacter et al., 2015).

Según el estudio de Haltigan y Vaillancourt (2014), los niños que no están diagnosticados y sin embargo presentan síntomas relativos al TDAH, como problemas de atención e hiperactividad, presentan mayor probabilidad de ser víctimas de acoso escolar.

Por último, el tener miedo a la victimización se asociada a mayor probabilidad de ser acosado (Kollerova y Smolik, 2016), al igual que ser poco sociable (Cillessen y Lansu, 2015), y el tener unos altos niveles de ansiedad (Berger-Silva, 2012; Bowes et al., 2013; Haltigan y Vaillancourt, 2014; Kärnä, et al., 2010; Moses y Williford, 2017; Saarento et al., 2013). No obstante, en esta última, la probabilidad aumenta tanto para acosado como para acosador (Jansen et al., 2011). Otro indicativo de acoso es la autoeficacia emocional: puntuar bajo en esta variable aumenta la probabilidad de ser víctima de acoso en la red (Eden, Heiman, y Olenik-Shemesh, 2016). Por el contrario, puntuar alto en satisfacción general de la vida y bienestar personal ejerce como factor protector de victimización (Alcántara et al., 2017).

- Problemas externalizados.

Puntuar alto en externalización de problemas se asocia a victimización (Bowes et al., 2013; Cillessen y Lansu, 2015; Foster y Books-Gunn, 2013; Watt et al., 2015). El problema externalizado más común es la agresividad y la mayoría de los estudios 
que la analizan hallan que esta es una variable predictora de victimización (Jansen et al., 2011; Moses y Williford, 2017). No obstante, en algunos estudios esta variable es factor de riesgo de ser acosador y no acosado (Sugimura et al., 2017). Los hábitos de molestar, hablar alto o mascar fuerte (Guerra, Williams, y Sadek, 2011 ), el escaso autocontrol (Atik y Oya, 2013; Verlinde et al., 2014) y ser mentiroso (Guerra, Williams, y Sadek, 2011) también se asocian a una mayor probabilidad de ser víctima de acoso escolar. Por el contrario, las actitudes prosociales son factor protector frente a la victimización (Berger-Silva, 2012; Griese y Bush, 2014; Sugimura et al., 2017).

\section{FACTORES ESCOLARES}

\section{COMPROMISO ACADÉMICO}

El rendimiento académico es predictor de ser víctima de acoso. Sobre todo se ha establecido relación con bajo rendimiento, encontrando de manera consistente que este es factor de riesgo de ser víctima (Atik y Güneri, 2013; Berger-Silva, 2012; Bowes et al., 2013; Cillessen y Lansu, 2015; Garaigordobil y Machimbarrena, 2017; Glew et al., 2008; Wandera et al., 2017). No obstante, algunos estudios han relacionado el alto rendimiento académico con una mayor probabilidad de ser acosador y/o victima (Goldbach, Sterzing, y Stuart, 2017). La asistencia al colegio también se ha analizado como un indicador de acoso: el alumnado que no va al centro con regularidad tiene mayores posibilidades de estar involucrado en algún tipo de rol dentro del bullying (Goldbach, Sterzing, y Stuart, 2017).

\section{RELACIÓN CON OTROS ESTUDIANTES}

De manera consistente, una baja popularidad (Andrews et al., 2016; BergerSilva, 2012; Wolke, Woods, y Samara, 2009), un escaso apoyo social (Alcántara et al., 2017; Burns et al., 2008; Eden, Heiman, y Olenik-Shemesh, 2016; Griese y Bush, 2014; Han, Zhang y Zhang, 2017;
Kollerova y Smolik, 2016; Pouwelse et al., 2011 ) y el rechazo entre el alumnado (Kärnä, et al., 2010; Kollerova y Smolik, 2016; Sainio et al., 2012) se asocia a una mayor probabilidad de ser víctima. En el caso de las aulas grandes esta correlación es más fuerte (Saarento et al., 2013). Eden, Heiman y Olenik-Shemesh, (2016), además, hallan relación entre puntuar alto en soledad o bajo en autoeficacia social y ser acosado en la red. Por el contrario, contar con apoyo social en el aula constituye un factor protector contra el acoso. Tener un hermano (gemelo) en clase disminuye la probabilidad de ser víctima, sobre todo si son dos hermanas (Veldkamp et al., 2017). Tener amigos en la escuela constituye un factor protector, salvo que esos amigos sean víctimas de bullying, lo cual constituye un factor de riesgo (Echols y Graham, 2016). Una minoría de estudios advierten que puntuar alto en popularidad también se asocia a ser víctima de acoso escolar (Andrew et al., 2016).

\section{RELACIÓN PREVIA CON EL ACOSO}

Ser acosador aumenta la probabilidad de ser víctima de acoso (Burns et al., 2008), si bien esta asociación es más fuerte a la inversa (Haltigan y Vaillancourt, 2014). Por otro lado, ser víctima es predictor de seguir siéndolo en el futuro (Serdiouk et al., 2015; Wolke, Woods, y Samara, 2009) y de disminución de las relaciones entre pares futuras (Leadbeater et al., 2015).

\section{EL CLIMA DE CONVIVENCIA EN LA ESCUELA}

La percepción de un clima escolar malo (Guerra, Williams, y Sadek, 2011 ; Wang et al., 2014) y poco seguro (Gage, Prykanowski, y Larson, 2014; Glew et al., 2008) se asocia a una mayor probabilidad de ser víctima. En las escuelas en las que los alumnos respetan menos las diferentes culturas hay mayores tasas de victimización (Gage, Prykanowski, y Larson, 2014). Además, que los compañeros refuercen el acoso también constituye un factor de riesgo (Kärnä, et al., 2010). Las 
aulas con mucha jerarquía se asocian a una mayor probabilidad de que existan víctimas de tipo relacional (Wolke, Wood, y Samara, 2009) y de sufrir acoso en el futuro (Serdiouk et al., 2015).

\section{SATISFACCIÓN EN LA ESCUELA}

No sentirse integrado en a la escuela correlaciona positivamente con ser acosado (Glew et al., 2008), al igual que cambiar de escuela (Wolke, Wood, y Samara, 2009) - puntuar bajo en ajuste escolar (García, Romera, y Ortega, 2015). Por el contrario, las actitudes en el grupo de defender a las víctimas constituyen un factor protector ante la victimización (Saarento et al., 2013). En cuanto a la satisfacción referida a los maestros, las actitudes contra la violencia por parte de los docentes percibida por el alumnado, se relaciona con una menor victimización (Saarento et al., 2013; Serdiouk et al., 2015), al igual que sentir su apoyo emocional (Lawson et al., 2013). Además, percibir un trato injusto (mal reparto de las oportunidades de participar en actividades escolares con materiales y equipo) por parte del profesorado conlleva también una mayor probabilidad de ser víctima (Leadbeater et al., 2015).

\section{CARACTERÍSTICAS DEL CENTRO EDUCATIVO}

En cuanto a la titularidad del centro, algunos estudios han hallado que ser alumno de un centro público aumenta la probabilidad de ser acosado (Foster y Brooks-Gunn, 2013). Otros han hallado que serlo de un centro de élite aumenta la probabilidad de ser acosado y/o acosador (Han, Zhang, Zhang, 2017). Por otro lado, cuanto mayor sea la ratio alumnoprofesor del aula, mayor será la probabilidad de ser victimizado (Bradshaw, Sawyer, y O’Brennan, 2009).

\section{FACTORES FAMILIARES}

VARIABLES SOCIOECONÓMICAS
La mayoría de los estudios que analizan estas variables concluyen que es más probable que un alumno sea víctima de acoso escolar si pertenece a una familia de bajo nivel socioeconómico (Bowes et al., 2013; Jansen et al., 2011 ; Jansen et al., 2012; Seo et al., 2017) y sus padres tienen un bajo nivel educativo (Jansen et al., 2012; Seo et al., 2017; Von y Petermann, 2010). No obstante, algunos estudios han hallado relación entre puntuar alto en nivel socioeconómico y ser acosado (Foster y Brooks-Gunn, 2013). Además, ser hijo de padres jóvenes se asocia a un mayor probabilidad de estar involucrado en bullying en cualquiera de sus roles (Jansen et al., 2012).

\section{ESTRUCTURA FAMILIAR}

Los trabajos revisados que estudian esta variable encuentra relación entre ser víctima de acoso y pertenecer a una familia monoparental (Jansen et al., 2012; Wandera et al., 2017). Por otro lado, tener estabilidad en el hogar ejerce también como factor protector ante el acoso escolar (Foster y Brooks-Gunn, 2013).

\section{APOYO FAMILIAR}

Mantener una relación poco afectiva entre padres e hijos aumenta el riesgo de ser víctima de bullying (Atik y Güneri, 2013; Brendgen et al., 2016; Liv et al., 2012; Seo et al., 2017). Del mismo modo, un escaso apoyo familiar se relaciona con ser víctima de intimidación en la escuela (Alcántara et al., 2017; Brendgen et al., 2016; Gage, Prykanowski, y Larson, 2014). Algunos trabajos destacan especialmente la importancia del apoyo de la madre (Alcántara et al., 2017). En relación al apoyo familiar dentro de la escuela, los hijos de los padres que no se involucran con el centro escolar son niños más acosados (Leadbeater et al, 2015).

\section{ESTILO EDUCATIVO DE LOS PADRES}

Tanto la falta como el exceso de control son factores de riesgo de ser víctima de acoso. Los hijos de padres permisivos 
(Garaigordobil y Machimbarrena, 2017) y poco supervisores (Atik y Güneri, 2013) es más probable que sean acosados. También los hijos de padres autoritarios (Cerezo et al., 2018; Garaigordobil y Machimbarrena, 2017) o sobreprotectores (Garaigordobil y Machimbarrena, 2017). Además, los hijos de padres inadaptados y que han recibido una crianza deficitaria se asocian también a mayor posibilidad de ser víctimas de acoso escolar (Lereya y Wolke, 2013).

\section{EXPOSICIÓN A LA VIOLENCIA EN LA FAMILIA}

Los niños maltratados por sus padres son más propensos a ser agredidos en la escuela (Bowes et al., 2013; Foster y Brooks-Gunn, 2013). También ser testigo de violencia dentro del hogar es un predictor de ser víctima de acoso (Ameli et al., 2017; Lereya y Wolke, 2013; Wandera et al., 2017).

\section{SALUD MENTAL DE LOS PADRES}

Los hijos de padres con problemas mentales son más propensos a estar involucrados en cualquiera de los roles del acoso (Jansen et al., 2011). Algunos estudios analizan más específicamente el efecto de problemas mentales de la madre durante y después del embarazo: cuando existen, el hijo tendrá más posibilidades de ser victimizado (Lereya y Wolke, 2013). Tener padres con estrés parental también aumenta la posibilidad de ser víctima de acoso escolar y en la red (Garaigordobil y Machimbarrena, 2017).

\section{FACTORES COMUNITARIOS}

\section{ASPECTOS RELACIONADOS CON EL $B A R R I O$}

Tener pocos amigos que sirvan de apoyo en el barrio también supone un factor de riesgo (Alcántara et al., 2017). Vivir en un barrio con bajo nivel socioeconómico aumenta la probabilidad de ser víctima de acoso escolar (Foster y Brooks-Gunn, 2013). Pertenecer a escuelas de zonas pobres se asocia a mayor posibilidad de ser víctima y/o acosador (Jansen et al., 2012). En Brasil, estar matriculado en una escuela estatal - rural eleva la probabilidad de ser víctima (Alcántara et al., 2017). En EEUU, acudir a escuelas suburbanas aumenta la probabilidad de ser víctima (Bradshaw, Sawyer y O’Brennan, 2009).

\section{RELACIÓN CON LOS MEDIOS DE COMUNICACIÓN Y LOS MEDIOS DE ENTRETENIMIENTO}

Sólo una de las publicaciones analizadas ha estudiado la capacidad predictiva de esta variable. Según Watt et al. (2015), cuanto mayor sea el tiempo que se ve la televisión en preescolar mayor será la probabilidad de ser víctima de acoso en Primaria.

\section{DISCUSIÓN}

El objetivo de este estudio ha sido identificar los principales predictores de ser víctima de acoso escolar en Educación Primaria, a partir de una revisión sistemática de los artículos empíricos más relevantes publicados en la última década. Los resultados obtenidos permiten advertir algunas tendencias.

En relación a las variables sociodemográficas, los niños son más propensos a ser víctimas de acoso escolar que las niñas. Esto puede deberse a ellos son más agresivos e impulsivos que ellas y, por tanto, se ven involucrados en más conflictos y reciben menos apoyo social (Ikiza y Cakar 2010; Pastor et al., 2012). Además, el alumnado más joven tiene mayor probabilidad de ser víctima de bullying ya que cuanto menor es la edad de los niños, menor es su desarrollo físico, psicosocial y académico, lo que les hace ser más vulnerables ante el acosado escolar (Craig et al., 2009; Smith et al., 1999).

Sobre los factores psicológicos, las tendencias más destacadas se han dado en la internalización y externalización de problemas. La asociación entre problemas internalizados o externalizados y ser víctima de acoso escolar es muy consistente en anteriores estudios (Cook et al., 2010). En 
referencia a los problemas internalizados, la ansiedad y los síntomas depresivos son las variables más estudiadas y ambas son factores de riesgo de ser acosado en la escuela (Cook et al., 2010). En relación a los problemas externalizados, la agresividad, los hábitos de molestar y ser mentiroso constituyen factores de riesgo de ser acosado, mientras que las actitudes prosociales han sido consideradas como factor protector ante el acoso (Griese y Bush, 2014).

En cuanto a los factores escolares, el rendimiento académico es la variable que mayor interés suscita. Todos los estudios revisados que la han analizado, salvo uno, han hallado relación entre puntuar bajo en rendimiento académico y ser víctima de acoso en la escuela. Con respecto a la relación con los compañeros, tanto puntuar bajo en popularidad como en apoyo social son factores de riesgo de ser agredido en el centro escolar. La ausencia de apoyos sociales es una de las variables que facilita que ocurra el acoso (Cava et al., 2010).

En referencia a los factores familiares, las tendencias más destacadas se centran en varios aspectos. En primer lugar, tanto un bajo nivel socioeconómico como educativo de los padres son predictores de ser víctima de acoso. Los niños que pertenecen a este tipo de familias tienen menos acceso a recursos intelectuales y sociales (Jansen et al., 2012). En segundo lugar, una baja relación afectiva y un escaso apoyo familiar son factores de riesgo para ser intimidados por los compañeros ya que cuando, por el contrario, las victimas reciben afecto y apoyo por parte de su familia tienen mayores recursos emocionales (Bowes, et al., 2010). Y en tercer lugar, ser testigo de violencia dentro de la familia también aumenta la probabilidad de ser víctima de bullying, puesto que estos niños tienen una actitud de aceptación a la violencia, lo que les hace más vulnerables ante ella (Bowes et al., 2009).

La presente revisión sistemática supone una aportación al campo de estudio. Aporta una visión actualizada de la última década sobre los factores de protección y riesgo de ser víctima de acoso escolar en Educación
Primaria. Además, la mayoría de las revisiones sistemáticas anteriormente publicadas se centran en Educación Secundaria, y en algunos casos junto a la Educación Primaria. Sin embargo, no se han encontrado revisiones relativas al tema de estudio centradas únicamente en Educación Primaria.

Este trabajo ofrece importantes implicaciones prácticas: conocer los factores de riesgo y protección de padecer bullying es el paso previo necesario para diseñar programas preventivos eficaces. No obstante, el presente trabajo también tiene sus limitaciones. Una de ellas, es que se ha acotado la búsqueda a unas bases de publicaciones específicas, por lo que pueden existir otros estudios relevantes no contemplados en esta revisión. Una segunda limitación es que los resultados de las investigaciones aquí revisadas pueden estar influenciadas por el país o contexto de donde fue extraída la muestra y no ser directamente extrapolables a otros contextos. En referencia a las futuras líneas de investigación, se recomienda ampliar las bases de publicaciones y analizar el posible impacto del sesgo de publicación.

\section{- Agradecimientos}

Este trabajo ha sido financiado por el Gobierno del Principado de Asturias (Plan de Ciencia, Tecnología e Innovación 20182022) y la Unión Europea (Fondo Europeo de Desarrollo Regional - FEDER) (Ref. FCGRUPINIDI/2018/000199); por la Consejería de Educación del Principado de Asturias (ayuda "Severo Ochoa" a la primera autora); y por el Ministerio de Ciencia, Innovación y Universidades del Gobierno de España (Ref. PGC2018-097739-B-100).

- Conflicto de intereses.

Los autores declaran no tener ningún conflicto de intereses.

\section{REFERENCIAS}

*Artículos que conforman la muestra de publicaciones revisada.

*Alcántara, S. C., González-Carrasco, M., Montserrat, C., Viñas, F., Casas, F., \& Abreu, D. P. (2017). Peer violence in the School Environment and Its Relationship with Subjective Well-Being and Perceived Social Support Among Children and Adolescents 
in Northeastern Brazil. Journal of Happiness Studies, 18(5), 1507-1532. https://doi. org/10.1007/s10902-016-9786-1.

Álvarez-García, D., García, T., \& Núñez, J. C. (2015). Predictors of school bullying perpetration in adolescence: A systematic review. Aggression and Violent Behavior, 23, 126-136. http://doi.org/10.1016/i. avb.2015.05.007

*Ameli, V., Meinck, F., Munthali, A., Ushie, B., \& Langhaug, L. (2017). Associations between adolescent experiences of violence in Malawi and gender-based attitudes internalizing and externalizing behaviors. Child Abuse \& Neglect, 67, 305-14. https:// doi.org/10.1016/i.chiabu.2017.02.027.

*Andrews, N. C. Z., Hanish, L. D., Updegraff, K. A., Martin, C. L., \& Santos, C. E. (2016). Targeted victimization: Exploring linear and curvilinear associations between social network prestige and victimization. Journal of Youth and Adolescence, 45, 1772-1785. https://doi.org/10.1007/s10964-0160450-1.

*Atik, G., \& Güneri, O. Y. (2013). Bullying and victimization: Predictive role of individual, parental, and academic factors. School Psychology International, 34(6), 658-673. http://doi. org/10.1177/0143034313479699.

*Berger-Silva, C. (2012). Trajectories of school victimization: Characteristics and risk factors in chilean teenagers. Universitas Psychologica, $17(1), 103-118$.

Bowes, L., Arseneault, L., Maughan, B., Taylor, A., Caspi, A., \& Moffitt, T. E. (2009). School, neighborhood, and family factors are associated with children's bullyinginvolvement: A nationally representative longitudinal study. Journal of the American Academy of Child \&Adolescent Psychiatry, 48(5), 545-553. http://doi. org/10.1097/CHI.0b013e31819cb017.

Bowes, L., Maughan, B., Caspi, A., Moffitt, T. E., \& Arseneault, L. (2010). Families promote emotionaland behavioural resilience to bullying: Evidence of an environmental effect. Journal of Child Psychol-ogy and Psychiatry, 51 (7), 809-817.

*Bowes, L., Maughan, B., Ball., H, Shakoor,
S., Ouellet-Morin, I., Caspi, A., Moffit, T. E., \& Arseneault, T. (2013). Chronic bullying victimization across school transitions: the role of genetic and environmental influences. Development Psychopathology, 25(2), 333-46. https://doi.org/10.1017/ S0954579412001095.

*Bradshaw, C. P., Sawyer, L. A., \& O'Brennan, L. M. (2009). A social disorganization perspective on bullying-related attitudes and behaviors: The influence of school context. American Journal of Community Psychology, 43(3-4), 204-220. https://doi. org/10.1007/s 10464-009-9240-1.

*Brendgen, M., Girard, A., Vitaro, F., Dionne, G., \& Boivin, M. (2016). Personal and familial predictors of peer victimization trajectories from primary to secondary school. Developmental Psychology, 52(7), 1103-1114. https://doi.org/10.1037/ dev0000107.

Brunstein, A., Sourander, A., \& Gould M. (2010). The association of suicide and bullying in childhood to young adulthood: a review of cross-sectional and longitudinal research findings. The Canadian Journal of Psychiatry, 55(5), 282-288.

*Burns S. K., Cross D., Alfonso H., \& Maycock B. R. (2008). Predictors of bullying among 10 to 11 year old school students in Australia. Advances in School Mental Health Promotion, 1 (2), 49-60. https://doi. org/10.1080/1754730X.2008.9715728.

Cava, M. J., Buelga, S., Musitu, G., \& Murgui, S. (2010). Violencia escolar entre adolescentes y sus implicaciones en el ajuste psicosocial: un estudio longitudinal. Revista de Psicodidáctica, 15, 21-34.

${ }^{*}$ Cerezo, F., Ruiz-Esteban, C., Sanchez-Lacasa C., \& Gonzalo, J. J. A. (2018). Dimensions of parenting styles, social climate, and bullying victims in primary and secondary education. Psicothema, 30(1), 59-65. https://doi. org/10.7334/psicothema2016.360.

${ }^{*}$ Cillessen, A. H., \& Lansu, T. A. (2015). Stability, correlates, and time-covarying associations of peer victimization from grade 4 to 12 . Journal of Clinical Child \& Adolescent Psychology, 44(3), 456-470. https://doi.org /10.1080/15374416.2014.958841. 
Cook, C. R., Williams, K. R., Guerra, N. G., Kim, T. E., \& Sadek, S. (2010). Predictors of bullying and victimization in childhood and adolescence: A meta-analytic investigation. School Psychology Quarterly, 25(2), 65-83.

Craig, W., Harel-Fisch, Y., Fogel-Grinvald, H., Dostaler, S., Hetland, J., SimonsMorton, B., Molcho, M., Gaspar de Mato, M., Overpeck, M., Due, P., \& Pickett, W. (2009). A cross-national profile of bullying and victimization among adolescents in 40 countries. International Journal of Public Health, 54, 209-215.

*Echols, L., \& Graham, S. (2016). For Better or Worse: Friendship Choices and Peer Victimization Among Ethnically Diverse Youth in the First Year of Middle School. Journal of Youth and Adolescence, 45(9), 1862 76. https://doi.org/10.1007/s10964-0160516-0.

*Eden, S., Heiman, T., \& Olenik-Shemesh, D. (2016). Bully versus victim on the internet: The correlation with emotional-social characteristics. Education and Information Technologies, 21 (3), 699-713. https://doi. org/10.1007/s10639-014-9348-2.

Espelage, D. L. (2014). Ecological Theory: Preventing Youth Bullying, Aggression, and Victimization. Theory into Practice, 53(4), 257-64.

Estévez, E., Murgui, S., \& Musitu, G. (2009). Psychological adjusment in bullies and victims of school violence. European Journal of Psychology of Education, 4, 473-483.

*Foster, H., \& Brooks-Gunn, J. (2013). Neighborhood, Family and Individual Influences on School Physical Victimization. Journal of Youth and Adolescence, 42(10), 1596-1610. https://doi.org/10.1007/ s10964-012-9890-4.

*Gage, N. A., Prykanowski, D. A., \& Larson, A. (2014). School Climate and Bullying Victimization: A Latent Class Growth Model Analysis. School Psychology Quarterly, 29(3), 256-71. https://doi.org/10.1037/ spq0000064.

*Garaigordobil, M., \& Machimbarrena, J. M. (2017). Stress, competence, and parental educational styles in victims and aggressors of bullying and cyberbullying. Psicothema,
29(3), 335-340. https://doi.org/10.7334/ psicothema2016.258.

Garcia-Bacete, F. J, Sureda, I., \& Monjas, M. I. (2010). El rechazo entre iguales en la educación primaria: una panorámica general. Anales de psicología, 26(1), 123136

*García, C. M., Romera, E. M., \& Ortega, R. (2015). Explicative factors of face-toface harassment and cyberbullying in a sample of primary students. Psicothema, 27, 347-353. https://doi.org/10.7334/ psicothema2015.35.

*Glew, G. M., Fan, M. Y., Katon, W., \& Rivara, F. P. (2008). Bullying and school safety. Journal of Pediatrics, 152(1), 123-128. https://doi. org/10.1016/i.jpeds.2007.05.045.

*Goldbach, J. T., Sterzing, P. R., \& Stuart M. J. (2017). Challenging Conventions of Bullying Thresholds: Exploring Differences between Low and High Levels of Bully-Only, Victim-Only, and Bully-Victim Roles. Journal of youth and adolescence, 47(3), 586-600. https://doi.org/10.1007/s10964-017-

\section{5-4.}

Graham, S., Bellmore, A. D., \& Mize, J. (2006). Peer victimization, aggression, and their coocurrence in middle school: pathways to adjustment problems. Journal of Abnormal Child Psychology, 34(3), 363-378.

*Griese, E. R.., \& Buhs, E. S. (2014). Prosocial Behavior as a Protective Factor for Children's Peer Victimization. Journal of Youth and Adolescence, 43(7), 1052-1065. https:// doi.org/10.1007/s 10964-013-0046-y.

*Guerra, N., Williams, K. R., \& Sadek, S. (2011). Understanding bullying and victimization during childhood and adolescence: A mixed methods study. Child Development, 82(1), 295-310. https://doi.org/10.1111/i.1467. 8624.2010.01556.x.

*Haltigan, J. D., \& Vaillancourt, T. (2014). Joint Trajectories of Bullying and Peer Victimization Across Elementary and Middle School and Associations With Symptoms of Psychopathology. Developmental Psychology, 50(11), 2426-2436. https:// doi.org/10.1037/a0038030.

*Han, Z., Zhang, G., \& Zhang, H. (2017). School bullying in urban China: Prevalence 
and correlation with school climate. International Journal of Environmental Research and Public Health, 14(10), 1116. https://doi.org/10.3390/ijerph 14101116.

Hymel, S., \& Swearer, S. M. (2015). Four Decades of Research on School Bullying. American Psychologist, 70(4), 293-299. https://doi.org/10.1037/a0038928

Ikiza, F. E., \& Cakar, F. S. (2010). Perceived social support and self-esteem in adolescence. Procedia Social BehavioralSciences, 5, 2338-2342.

Jankauskiene, R., Kardelis, K., Sukys, S., \& Kardeliene, L. (2008). Associations between school bullying and psychosocial factors. Social Behavior \& Personality, 36(2), 145 162.

*Jansen, D. E., Veenstra, R., Ormel, J., Verhulst, F.C., \& Reiineveld, S. A. (2011). Early risk factors for being a bully, victim, or bully/ victim in late elementary and early secondary education. The longitudinal TRAILS study. BMC Public Health, 11-440. https://doi. org/10.1186/1471-2458-11-440.

*Jansen, P. W., Verlinden, M., Dommisse-van Berkel, A., Mieloo, C., van der Ende, J., Veenstra, R., Verhulst, F. C., Jansen, W., \& Tiemeier, H. (2012). Prevalence of bullying and victimization among children in early elementary school: Do family and school neighbourhood socioeconomic status matter? BMC Public Health, 12. https://doi. org/10.1186/1471-2458-12-494

*Kärnä, A., Voeten, M., Poskiparta, E., \& Salmivalli, C. (2010). Vulnerable children in varying classroom contexts: Bystanders' behaviors moderate the effects of risk factors on victimization. Merrill-Palmer QuarterlyJournal of Developmental Psychology, 56(3), 261-282.

Kerr, J. C., Valois, R. F., Huebner, E. S., \& Drane, J. W. (2011). Life satisfaction and peer victimization among USA public high schools adolescents. Child Indicators Research, 4, 127-144.

Kljakovic, M., \& Hunt, C. (2016). A metaanalysis of predictors of bullying and victimisation in adolescence. Journal of Adolescence, 49, 134-45.

*Kollerova, L., \& Smolik, F. (2016). Victimization and its associations with peer rejection and fear of victimization: Moderating effects of individual-level and classroom-level characteristics. British Journal of Educational Psychology, 86(4), 640-56. https://doi. org/10.1111/bjep.12129.

*Lawson, M. A., Alameda-Lawson, T., Downer, J., \& Anderson, E. (2013). Analyzing subpopulation profiles and risk factors for school bullying. Children and Youth Services Review, 35(6), 973-983. https://doi. $\mathrm{org} / 10.1016 /$ i.childyouth.2013.03.006

*Leadbeater, B., Sukhawathanakul, P., Smith, D., \& Bowen, F. (2015). Reciprocal associations between interpersonal and values dimensions of school climate and peer victimization in elementary school children. Journal of Clinical Child and Adolescent Psychology, 44(3), 480-493. https://doi.org/10.1080/ 15374416.2013 .873985$.

*Lee S., Oh, I., \& Lee, J. (2014). Psychosocial Characteristics of Bullies, Victims, BullyVictims among Elementary School Students. Korean Journal of Youth Studies, 21 (5), 391 416.

*Lereya, S. T., \& Wolke, D. (2013). Prenatal family adversity and maternal mental health and vulnerability to peer victimisation at school. Journal of Child Psychology and Psychiatry and Allied Disciplines, 54(6), 644652. https://doi.org/10.1111/icpp. 12012. *Liu, X., Xin-hua, Y., Li-hua, Z., Li-jun, W., \& Lin-yan, S. (2012). Relationship between attachment to parents and bullying, victimization of 4-6th graders. Chinese Journal of Clinical Psychology, 20(2), 24648.

López, R., Amaral, A. F., Ferreira, J., \& Barroso, T. (2011). Factors related to the bullying phenomenon in school context: Integrative literature review. Revista de Enfermagem Referência, 3(5), 153-162.

*Lumeng, J. C., Forrest, P., Appugliese, D. P., Kaciroti, N., Corwyn, R. F., \& Bradley, R. H. (2010). Weight status as a predictor of being bullied in third through sixth grades. Pediatrics, 125(6), 301-307. https://doi. org/10.1542/peds.2009-0774.

*Moses, M., \& Williford, A. (2017). Individual indicators of self-reported victimization 
among elementary school-age students: A latent class analysis. Children and Youth Services Review, 83, 33-40. https://doi. org/10.1016/i.childyouth.2017.10.006.

Olweus, D. (1993). Bullying at school: What we know and what we can do. Malden, MA: Blackwell Publishing.

Pastor, Y., Quiles, Y., \& Pamies, L. (2012). Apoyo social en la adolescencia: adaptación y propiedades psicométricas del 'Social Support Scale for Children' de Harter (1985). Revista de Psicología Social, 27(1), 39-53.

*Pouwelse, M., Bolman, C., Lodewijkx, H., \& Spaa, M. (2011). Gender differences and social support: Mediators or moderators between peer victimisation and depressive feelings? Psychology in the Schools, 48(8), 800-814. https://doi.org/10.1002/ pits.20589.

Rettew, D. C., \& Pawlowski S. (2016). Bullying. Child and Adolescent Psychiatric Clinics of North America, 25(2), 235-242.

*Saarento, S., Kärnä, A., Hodges, E. V. E., \& Salmivalli, C. (2013). Student-, classroom-, and school-level risk factors for victimization. Journal of School Psychology, 51, 421-434. https://doi.org/10.1016/i. jsp.2013.02.002.

Saarento, S., Garandeau, C. F., \& Salmivalli, C. (2014). Classroom- and school-level contributions to bullying and victimization: A review. Journal of Community \& Applied Social Psychology, 25(3), 204-218. https:// doi.org/10.1002/casp.2207.

*Sainio, M., Veenstra, R., Huitsing, G., \& Salmivalli, C. (2012). Same- and othersex victimization: Are risk factors similar? Aggressive Behavior, 38(6), 442-455. https://doi.org/10.1002/ab.21445.

*Schacter, H. L., White, S. J., Chang, V. Y., \& Juvonen, J. (2015). "Why me?": Characterological selfblame and continued victimization in the first year of middle school. Journal of Clinical Child \& Adolescent Psychology, 44(3), 446-455. https://doi.or $\mathrm{g} / 10.1080 / 15374416.2013 .865194$.

*Seo, H., Young-Eun, J., Moon-Doo, K., \& Won-Myong, B. (2017). Factors associated with bullying victimization among Korean adolescents. Neuropsychiatric Disease and Treatment, 13, 2429-35. https://doi. org/10.2147/NDT.S140535.

*Serdiouk, M., Rodkin, P., Madill, R., Logis, H. , \& Gest, S. (2015). Rejection and victimization among elementary school children: The buffering role of classroom-level predictors. Journal of Abnormal Child Psychology, 43(1), 5-17. https://doi.org/10.1007/s10802013-9826-9.

Smith, P. K., Madsen, K. C., \& Moody, J. C. (1999). What causes the age decline in reports of being bullied at school? Towards a developmental analysis of risks of being bullied. Educational Research, 41, 267285.

*Sugimura, N., Troop-Gordon, W., Berry, D., \& Rudolph, K. D. (2017). Early Social Behaviors and the Trajectory of Peer Victimization Across the School Years. Developmental Psycholog, 53(8), 1447-1461. https://doi. org/10.1037/dev0000346.

*Veldkamp, S. A., Van Bergen, E., De Zeeuw, E. L., Van Beijsterveldt, C. E., Boomsma, D. I., \& Bartels, M. (2017). Bullying and victimization: The effect of close companionship. Twin Research and Human Genetics, 20(1), 1927. https://doi.org/10.1017/thg.2016.99. *Verlinden, M., Veenstra, R., Ghassabian, A., Jansen, P. W., Hofman, A., Jaddoe, V. W. V., Verhulst, C. F., \& Tiemeier, H. (2014). Executive functioning and non-verbal intelligence as predictors of bullying in early elementary school. Journal of Abnormal Child Psychology, 42(6), 953-966. https:// doi.org/10.1007/s 10802-013-9832-y.

*Von Marées, N., \& Petermann, F. (2010). Bullying in German primary schools: Gender differences, age trends and influence of parents' migration and educational backgrounds. School Psychology International, 37 (2), 178-198. https://doi. org/10.1177/0143034309352416.

*Wandera, S. O., Clarke, K., Knight, L., Allen, E., Walakira, E., Namy, S., Naker, D., \& Devries, K. (2017). Violence against children perpetrated by peers: A crosssectional school-based survey in Uganda. Child Abuse \& Neglect, 68, 65-73. https:// doi.org/10.1016/i.chiabu.2017.04.006.

*Wang, W., Vaillancourt, T., Brittain, H. L., 
McDougall, P., Krygsman, A., Smith, D., \& Hymel, S. (2014). School climate, peer victimization, and academic achievement: Results from a multiinformant study. School Psychology Quarterly, 29(3), 360-377. https://doi.org/10.1037/spq0000084.

*Watt, E., Fitzpatrick, L., Derevensky, C. J., \& Pagani, L. S. (2015). Too Much Television? Prospective Associations Between Early Childhood Televiewing and Later Self-reports of Victimization by Sixth Grade Classmates. Journal of Developmental and Behavioral Pediatrics, 36(6), 426-433. https://doi. org/10.1097/DBP.0000000000000186.

*Williford, A., Boulton, A. J., \& Jenson, J. M. (2014). Transitions between subclasses of bullying and victimization when entering middle school. Aggressive Behavior, 40(1), 24-41. https://doi.org/10.1002/ ab.21503.

*Wolke, D., Woods, S., \& Samara, M. (2009). Who escapes or remains a victim of bullying in primary school? British Journal of Developmental Psychology, 27(4), 835-851. https://doi. org/10.1348/026151008X383003.

Yang, S., Stewart, R., Kim, J., Kim, S., Shin, I., Dewey, M. E., Maskey, S., \& Yoon, J. S. (2013). Differences in predictorsof traditional and cyber-bullying: A 2-year longitudinal study in Korean school children. European Child \& Adolescent Psychiatry, 22, 309-318. http:// doi.org/10.1007/s00787-012-0374-6. 